\title{
THE PALINDROMIC WIDTH OF A FREE PRODUCT OF GROUPS
}

\author{
VALERY BARDAKOV ${ }^{凶}$ and VLADIMIR TOLSTYKH
}

(Received 12 November 2003; revised 19 December 2004)

Communicated by E. A. O'Brien

\begin{abstract}
Palindromes are those reduced words of free products of groups that coincide with their reverse words. We prove that a free product of groups $G$ has infinite palindromic width, provided that $G$ is not the free product of two cyclic groups of order two (Theorem 2.4). This means that there is no uniform bound $k$ such that every element of $G$ is a product of at most $k$ palindromes. Earlier, the similar fact was established for non-abelian free groups. The proof of Theorem 2.4 makes use of the ideas by Rhemtulla developed for the study of the widths of verbal subgroups of free products.
\end{abstract}

2000 Mathematics subject classification: primary 20E06; secondary $20 \mathrm{~F} 05$.

\section{Introduction}

Let $G$ be a group and $S$ a generating set of $G$ with $S^{-1}=S$. For each $x \in G$ define the length $l_{S}(x)$ of $x$ relative to $S$ to be the least $k$ such that $x$ is a product of $k$ elements of $S$. The supremum of the values $l_{S}(x)$ is called the width of $G$ with respect to $S$ and is denoted by wid $(G, S)$. Here, $\operatorname{wid}(G, S)$ is either a natural number, or $\infty$. In the case when wid $(G, S)$ is a natural number, every element of $G$ is a product of at most wid $(G, S)$ elements of $S$.

Generally speaking, the study of widths of a given group provides very useful information for understanding the structure of the group, and for various combinatorial, algorithmic and model-theoretic problems concerning the group. The concept of width provides a sort of 'measure' for generating sets: the greater the width of a group generated by a set $S$, the less 'massive' $S$ appears inside that group. With this point

The first author was supported by the RFFI, grant \# 02-01-01118. The second author was supported by a NATO PC-B grant via The Scientific and Technical Research Council of Turkey (TÜBITAK).

(C) 2006 Australian Mathematical Society 1446-7887/06 $\$ A 2.00+0.00$ 
of view, such phenomena as the finiteness (under certain natural conditions) of widths of all verbal subgroups of linear algebraic groups [6], the infiniteness of widths of verbal subgroups in various free constructions $[2,4,5,7]$, and the finiteness of the width of the symmetric group of an infinite set relative to any generating set [3] are of considerable importance.

The paper [1] examines the primitive and the palindromic widths of a non-abelian free group. Traditionally, attributes such as 'primitive' and 'palindromic' refer to the elements of the corresponding generating sets. Thus, for instance, the primitive width of a free group is its width relatively to the set of all primitive elements. Palindromic words or palindromes can be defined for all free products of groups (in particular, for free groups) as follows. Let

$$
G=\prod_{i \in I}^{*} G_{i}
$$

be a free product of groups. The palindromes associated with the free factorization (1.1) are those reduced words of $G$ that are read the same forward and backward. It is clear that $G$ is generated by palindromes. Then the palindromic width of $G$ is the width relative to the palindromes.

It is established in [1] that the palindromic width of any non-abelian free group is infinite. Moreover, the paper [1] contains a sketch of the proof of the infiniteness of the palindromic width of any free product of groups at least two of which are infinite.

In this paper we deal with arbitrary free products of groups. It turns out, that almost all free products have infinite palindromic width; the only exception is given by the free product of two cyclic groups of order two, when the palindromic width is two.

We would like to point out a certain similarity between the study of palindromic widths of free products that we are undertaking and the study of widths of verbal subgroups of free constructions. Indeed, like generators of a given verbal subgroup, all palindromic words are structurally alike. As the reader shall see this makes possible the application of the methods developed by Rhemtulla in [7] specially for the study of the widths of verbal subgroups of free products.

Let $G$ be a free product of groups. In Section 1 we consider the case where one of the free factors of $G$ contains elements of order at least three. We then use some of the functions introduced by Rhemulla in [7] to construct a so-called quasi-homomorphism, say, $\Delta_{1}$ whose values at palindromes are bounded by 2 . A function $\Delta: H \rightarrow \mathbb{Z}$, where $H$ is a group, is said to be a quasi-homomorphism, if for all $x, y \in H$

$$
\Delta(x y) \leq \Delta(x)+\Delta(y)+\text { const } .
$$

At the next step we show that $\Delta_{1}$ is not bounded from above. It then follows that $G$ has infinite palindromic width, since for each $k \in \mathbb{N}$ the values of $\Delta_{1}$ at words 
which are products of at most $k$ palindromes are uniformly bounded from above. The definition of $\Delta_{1}(g)$ is rather technical and we note that the value $\Delta_{1}(g)$, where $g \in G$ is a reduced word, reflects information on occurrences of subwords of the form $a \cdots a$ and $a^{-1} \cdots a^{-1}$ in the word $g$, where $a$ is a fixed element of order at least three from one of the free factors of $G$.

In Section 2 we study the case where there are no elements of order greater than two in the free factors of $G$, but there is a free factor that has at least two non-identity elements, say, $\boldsymbol{a}$ and $\boldsymbol{c}$. Then, like in Section 1, we construct a quasi-homomorphism $\Delta_{2}$ that vanishes at palindromes and is unbounded from above. This time, the value $\Delta_{2}(g)$ at a given element $g \in G$, reflects information on occurences of the subwords of the form $a \cdots c \ldots a$ in $g$.

\section{Case I: elements of order greater than or equal to 3 in one of the free factors}

Let

$$
G=\prod_{i \in I}^{*} G_{i}
$$

be a free product of groups. Any non-identity element $g$ of $G$ can be written as a reduced word

$$
g=v_{1} \cdots v_{n},
$$

where $v_{k}$ are elements of free factors $G_{i}$ and for every $k=1, \ldots, n-1$ the elements $v_{k}$ and $v_{k+1}$ lie in different free factors. The elements $v_{k}$ are said to be syllables of $g$. Conversely, if $v_{1}, \ldots, v_{n}$ are non-trivial elements from the free factors $G_{i}$ and for any $k$ the elements $v_{k}, v_{k+1}$ are members of different factors, then the product $v_{1} \cdots v_{n}$ is a non-identity element of $G$. The number of syllables of a reduced word $g \in G$ is called the length of $g$.

One may rewrite the syllables of $g$ in the reverse order, thereby obtaining the non-trivial element

$$
\bar{g}=v_{n} \cdots v_{1}
$$

of $G$. We call an element $g$ a palindrome associated with the free factorization (1.1) if $\bar{g}=g$. It is helpful to observe that each palindrome can be written in the form

$$
g=h v \bar{h}
$$

where $h$ is a reduced word and $v$ a syllable of $g$; so that $\bar{h}$ represents the reflection of $h$ relative to the central syllable $v$. 
Clearly, the elements of the free factors $G_{i}$ participating in a decomposition (1.1) are palindromes. Thus $G$ is generated by palindromes, and one can define the palindromic width of $G$ as the width of $G$ relative to the set of all palindromes.

As we noted in the introduction, the palindromic width of a free product is almost always infinite. We begin therefore with the description of the only exceptional case.

ClaIM 1.1. Let $G=A * B$ be a free product of cyclic groups of order two. Then the palindromic width of $G$ is two.

ProOF. Let $A=\langle a\rangle$ and $B=\langle b\rangle$. If a reduced word $g \in G$ begins with $a$, then

$$
g=(a b)^{k} \quad \text { or } \quad g=(a b)^{k} a
$$

for a suitable $k \in \mathbb{N}$. It is easy to see that any word of the form $(a b)^{k} a$ is a palindrome. Any word of the form $(a b)^{k}=(a b)^{k-1} a b$ is a product of two palindromes.

The bulk of this section is devoted to the proof of the infiniteness of palindromic width of a free product of non-trivial groups $G=A * B$ such that one of the groups, say, $A$, has elements of order at least three. In the next section we consider the case when both factors $A, B$ of the free product $A * B$ have no elements of order greater than or equal to 3 , but one of the factors has at least three elements. We shall see later that the general case can be easily reduced to the case of a free product of two groups.

In both of the described cases we shall actually be able to prove that the width of $G=A * B$ relative to some superset of the palindromes, consisting of palindromic-like words, is infinite. Clearly, this will imply the infiniteness of the palindromic width of $G$. With this idea in mind, we introduce the following definition.

Assume that $C$ is a subset of $A \cup B$. Consider the alphabet $X=C \cup\{\omega\}$, where the symbol $\omega$ belongs neither to $A$, nor to $B$. For every reduced word $g=v_{1} \cdots v_{n}$ of $G$, we define the word $\alpha(g)$ over the alphabet $X$ by replacing the syllables of $g$ that are not in $C$ by $\omega$. We then call the word $g$ a $C$-palindrome if $\alpha(g)$ is a palindrome over the alphabet $X$ (that is, $\alpha(g)$ 'reads the same backward and forward' as a word over the alphabet $X$.) It is worth mentioning that any palindrome is a $C$-palindrome for every $C \subseteq A \cup B$.

Applying the idea of representation (1.2) of palindromes, we see that a typical reduced $C$-palindrome looks like

$$
h v \tilde{h},
$$

where $v$ is a non-trivial element from $A \cup B, h$ is a reduced word of $G$, and $\tilde{h}$ denotes an arbitrary word which is, say, the result of a 'poor' reflection of the word $h$. That is, if $h=v_{1} \cdots v_{k}$, then $\widetilde{h}=v_{1}^{\prime} \cdots v_{n}^{\prime}$ and $v_{i} \in C$ implies that $v_{n-i+1}^{\prime}=v_{i}$. Let us stress that " $\sim$, denotes a one-placed predicate, not an operation, like the symbol ' - , 
PROPOSITION 1.2. Let $G=A * B$ be a free product non-trivial groups such that $A$ contains elements of order greater than or equal to 3 . Then the palindromic width of $G$ is infinite.

PROOF. Let us fix a non-identity element $a \in A$ of order greater than two. We have that $a^{-1} \neq \boldsymbol{a}$. We are going to find a quasi-homomorphism $\Delta_{1}: G \rightarrow \mathbb{Z}$ that takes reasonably small values at $\left\{a, a^{-1}\right\}$-palindromes (and consequently at palindromes).

Let $g=v_{1} \cdots v_{n}$ be a reduced word of $G$. Suppose that there are at least two occurrences of the aforesaid fixed element $\boldsymbol{a}$ in $g$. If $v_{i}=\boldsymbol{a}$ and $v_{j}=\boldsymbol{a}$ are consecutive occurrences of $\boldsymbol{a}$ in $g$, then we call the subword $v_{i+1} \cdots v_{j-1}$, an $\boldsymbol{a}$-segment of $g$ [7]. The length of this segment, the number $j-i-1$, is odd as the reader may easily see. The $a^{-1}$-segments of the reduced words are defined in a similar fashion.

For example, a word $\boldsymbol{a} b_{1} a_{2} b_{1} b_{3} \boldsymbol{a}^{-1} b_{4} a b_{5} \boldsymbol{a}^{-1}$ where $b_{i}$ are non-identity elements of $B$ and $a_{1} \in A \backslash\left\{a, a^{-1}\right\}$ contains two $a$-segments, that is, $b_{1}$ and $b_{2} a_{1} b_{3} a^{-1} b_{4}$ of length 1 and 5 respectively and one $a^{-1}$-segment of length 3 , namely, $b_{4} a b_{5}$.

For each $k \in \mathbb{N}$ we define the following two functions on the set of all reduced words of $G$ :

$$
d_{k}(g)=\text { the number of } \boldsymbol{a} \text {-segments of } g \text { of length } 2 k+1
$$

and

$$
d_{k}^{*}(g)=\text { the number of } a^{-1} \text {-segments of } g \text { of length } 2 k+1 .
$$

For every $k \in \mathbb{N}$, set also $t_{k}(g)=d_{k}(g)-d_{k}^{*}(g)$. The functions $d_{k}, d_{k}^{*}$ and $t_{k}$ are introduced in the paper [7] by Rhemtulla.

Clearly, for every reduced word $g \in G$

$$
d_{k}(g)=d_{k}^{*}\left(g^{-1}\right)
$$

and consequently

$$
t_{k}(g)+t_{k}\left(g^{-1}\right)=0 .
$$

LEMMA 1.3 ([7, Lemma 2.11]). The formula

$$
t_{k}(g h)=t_{k}(g)+t_{k}(h) \quad \forall g, h \in G
$$

holds for all but at most 9 natural numbers $k$.

Now we are ready to construct a desired quasi-homomorphism. Let $g$ be a reduced word from $G$ and let $\Delta_{l}(g)=\sum_{k=0}^{\infty} r_{k}$, where $r_{k} \geq 0$ is the remainder of division of the number $t_{k}(g)$ on 2 (only finitely many terms of the above series might be non-zero).

It follows immediately from Lemma 1.3 that

$$
\Delta_{1}(g h) \leq \Delta_{1}(h)+\Delta_{1}(h)+9 \quad \forall g, h \in G .
$$

Therefore, $\Delta_{1}$ is a quasi-homomorphism. 
LEMMA 1.4. If $g \in G$ is an $\left\{a, a^{-1}\right\}$-palindrome, then $\Delta_{1}(g) \leq 2$.

ProOF. By equation (1.3), a reduced word $g$ which is an $\left\{a, a^{-1}\right\}$-palindrome is $h v \widetilde{h}$ for some $h \in G$ and a non-trivial $v$ in $A \cup B$. Clearly, for all $k \in \mathbb{N}, d_{k}(h)=d_{k}(\widetilde{h})$. We claim therefore that for all but at most two $k \in \mathbb{N}, d_{k}(g)=2 d_{k}(h)$. Indeed, the 'new' $a$-segments that are not in $h$ or $\widetilde{h}$ can occur, if $g=u_{1} a u_{2} v \widetilde{u}_{2} a \widetilde{u}_{1}$, where $v \neq a$, and $a$ before $v$ indicates the last from the left occurrence of $a$ in $h$, or, if $g=u_{1} a u_{2} a \tilde{u}_{2} a \tilde{u}_{1}$. Assuming that $\left|u_{2} v \tilde{u}_{2}\right|=2 m+1$, we have in the first case that

$$
d_{m}(g)=2 d_{m}(h)+1 .
$$

In the second case, letting $k$ denote the natural number with $\left|u_{2}\right|=2 k+1$, we find

$$
d_{k}(g)=2 d_{k}(h)+2 .
$$

In particular, we see that for at most one natural $l$ the value $d_{l}(g)$ is odd. A similar argument applied to the $\boldsymbol{a}^{-1}$-segments of $g$ proves that for at most one $r \in \mathbb{N}$ the value of $d_{r}^{*}(g)$ is odd. Thus at most two of the values $t_{k}(g)$ might be odd, which completes the proof of the lemma.

Lemma 1.4 and equation (1.4) imply immediately that for every $g$ which is a product of at most $k\left\{\boldsymbol{a}, \boldsymbol{a}^{-1}\right\}$-palindromes (palindromes) $\Delta_{1}(g) \leq 11 k-9$.

Now we prove that $\Delta_{1}$ is not bounded from above. It suffices to find a sequence $\left(g_{n}\right)$ of elements of $G$ such that the sequence $\left(\Delta_{1}\left(g_{n}\right)\right)$ is infinitely increasing. For all natural numbers $n \geq 1$ let

$$
g_{n}=v w v w^{2} \cdots v w^{n} v,
$$

where $v=b \boldsymbol{a}$ and $w=b \boldsymbol{a}^{-1}$ and $b$ is a non-trivial element from $B$.

For every $n \geq 2$ we have

$$
g_{n}=g_{n-1}\left(b a^{-1}\right)^{n} b a=g_{n-1}^{\prime} a\left(b a^{-1}\right)^{n} b a .
$$

Thus $g_{n}$ gains only one 'new' $\boldsymbol{a}$-segment of length $2 n+1$ to be added to the $\boldsymbol{a}$-segments of $g_{n-1}$. This leads to

$$
\begin{aligned}
& d_{0}\left(g_{n}\right)=0, \\
& d_{1}\left(g_{n}\right)=\cdots=d_{n}\left(g_{n}\right)=1, \\
& d_{k}\left(g_{n}\right)=0 \quad \text { for all } k>n
\end{aligned}
$$

for all $n \geq 1$.

For every $n>1$, the word $g_{n}$ has $a^{-1}$-segments only of length 1 and 3 . Hence

$$
d_{k}^{*}\left(g_{n}\right)=0 \quad \text { for all } k \geq 2
$$


for all $n \geq 1$. Combining equations (1.5) and (1.6), we arrive at the inequality

$$
\Delta_{1}\left(g_{n}\right)=r_{0}+r_{1}+n-1 \geq n-1
$$

which is valid for all $n \geq 1$ (recall that $r_{k}$ is the remainder of the division of $t_{k}(g)$ on 2). Therefore, the sequence $\left(\Delta_{1}\left(g_{n}\right)\right)$ is infinitely increasing, as desired. The proposition is proven.

\section{Case II: two non-identity elements in one of the free factors}

We consider now the remaining case of free products of two groups.

Proposition 2.1. Let $G=A * B$ be a free product of groups, where $|A| \geq 3$ and $|B| \geq 2$ and both $A, B$ contain no elements of order greater than or equal to 3 . Then the palindromic width of $G$ is infinite.

Proof. It follows from the conditions that all non-identity elements of $A$ and $B$ are of order two. This implies that $A$ and $B$ are abelian, and hence $|A| \geq 4$. Thus we may pick up two distinct non-identity elements $a$ and $c$ from $A$.

We shall consider $\{\boldsymbol{a}, \boldsymbol{c}\}$-palindromes of $G$ and shall prove that the width of $G$ relative to those is infinite. This will imply the infiniteness of the palindromic width of $G$. We shall once again exploit ideas from Rhemtulla [7].

Since $a^{-1}$-segments are no longer useful, we work instead with $\boldsymbol{a}$-segments that contain a unique occurence of $c$; such an $a$-segment looks like

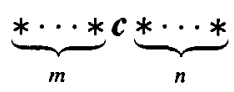

where there are no occurrences of neither $\boldsymbol{a}$, nor $c$ among syllables $*$. We call a segment (2.1) an a-segment of type $(m, n)$ if $m$ is the number of syllables before, and $n$ is the number of syllables after $c$.

The following functions on the set of reduced words of $G$ were introduced by Rhemtulla in [7]:

$$
\begin{aligned}
d_{m, n}(g) & =\text { the number of } a \text {-segments of type }(m, n) \text { in } g, \\
t_{m, n}(g) & =d_{m, n}(g)-d_{n, m}(g),
\end{aligned}
$$

where $m, n$ are arbitrary naturals.

LEMMA 2.2 ([7, page 581]). (i) For all pairs of natural numbers $(m, n)$ we have that the formulae

$$
d_{m, n}(g)=d_{n, m}\left(g^{-1}\right) \quad \text { and } \quad t_{m, n}(g)+t_{m, n}\left(g^{-1}\right)=0,
$$

hold for each reduced word $g$ in $G$; 
(ii) for all but at most 8 pairs $(m, n)$ of naturals we have that the formula

$$
t_{m, n}(g h)=t_{m, n}(g)+t_{m, n}(h)
$$

holds for each pair $g, h$ of reduced words of $G$.

This, as above, advises the following choice of a quasi-homomorphism to establish the infiniteness of the palindromic width:

$$
\Delta_{2}(g)=\sum_{0 \leq m<n} t_{m, n}(g)
$$

The mapping $\Delta_{2}$ is a quasi-homomorphism, since by Lemma 2.2

$$
\Delta_{2}(g h) \leq \Delta_{2}(g)+\Delta_{2}(h)+8
$$

for all $g, h \in G$.

LemMA 2.3. Assume $g$ is an $\{a, c\}$-palindrome. Then $\Delta_{2}(g)=0$.

Proof. By the general description (1.3), $g$ is of the form $h v \widetilde{h}$, where $v$ is a nonidentity element of $A \cup B$. Calculating the value of $\Delta_{2}$ at $g$ we are interested only in those $a$-segments of type $(m, n)$ for which $m$ is not equal $n$. Now

(1) if an $a$-segment of type $(m, n)$, say $a u_{1} c u_{2} a$, occurs in $h$, then its 'reflection' in $\widetilde{h}$ is $a \tilde{u}_{2} c \tilde{u}_{1} a$ and hence is of type $(n, m)$ and vice versa;

(2) the case, when the central element equals $a$ may bring a 'new' $a$-segment of type $(m, n)$, but also necessarily a 'new' segment of type $(n, m)$;

(3) finally, if the central element is $\boldsymbol{c}$, the only 'new' $\boldsymbol{a}$-segment of type $(m, n)$ that may occur in such a configuration is of type $(k, k)$ for some $k \in \mathbb{N}$.

Summing up, we see that for all pairs $(m, n)$ of naturals with $m<n$

$$
d_{m, n}(g)=d_{n, m}(g) \text {. }
$$

Hence $t_{m, n}(g)=0$ for all such pairs, and $\Delta_{2}(g)=0$.

Lemma 2.3 implies that for any word $g$ that is a product of at most $k\{a, c\}$-palindromes (palindromes), $\Delta_{2}(g) \leq 8 k-8$. Now we find a sequence $\left(g_{n}\right)$ of elements of $G$ with $\lim _{n \rightarrow \infty} \Delta_{2}\left(g_{n}\right)=\infty$. A non-trivial element $f$ which is not equal to $a, c$ can be found in $A$. Take also some non-trivial $b \in B$. For all $n \geq 1$, let

$$
g_{n}=v w v w^{2} v \cdots v w^{n} v,
$$

where $v=b \boldsymbol{a} b \boldsymbol{c}$ and $w=b f$. The element $g_{1}$, which is equal to

$$
v w v=b \boldsymbol{a} b \boldsymbol{c} b f \boldsymbol{b a} \boldsymbol{c}
$$


contains the unique $a$-segment of type $(m, n)$, where $m<n$, that is, the segment of type $(1,3)$. Thus $\Delta_{2}\left(g_{1}\right)=1$. Let $n>1$. We have

$$
g_{n}=g_{n-1} w^{n} v=g_{n-1}^{\prime} v w_{n} v=g_{n-1}^{\prime} b a b c(b f)^{n} b a b c .
$$

Then $g_{n}$ acquires the (only) 'new' $a$-segment of type $(1,2 n+1)$ to be added to the $a$-segments of $g_{n-1}$ of types $(1,2 k+1)$, where $k=1, \ldots, n-1$. This proves that $\Delta_{2}\left(g_{n}\right)=n$ for all $n \geq 1$. The proof of the proposition is now complete.

THEOREM 2.4. Let

$$
G=\prod_{i \in I}^{*} G_{i}
$$

be a free product of non-trivial groups. The palindromic width of $G$ with respect to the palindromes associated with the free factorization (2.2) is infinite if and only if at least one of the free factors has more than two elements or there are at least three free factors. The palindromic width of the free product of two cyclic groups of order two is two.

ProOF. The case of a free product of two groups is the subject of Claim 1.1, Proposition 1.2, and Proposition 2.1. Suppose now that $G$ is a free product of at least three groups. Let $i_{0}$ be an index from $I$. Set

$$
A=\prod_{i \neq i_{0}}^{*} G_{i} \text { and } B=G_{i_{0}} .
$$

We have that

$$
G=A * B
$$

and $G$ is a free product of two groups, one of which, $A$, is infinite. Then the width of $G$ relative to the palindromes associated with the free decomposition (2.3) is infinite. On the other hand, the set of all palindromes associated with (2.2) is contained in the set of all palindromes associated with (2.3). The width of $G$ with respect to the former set must therefore be infinite.

Acknowledgements The authors would like to thank Vladimir Shpilrain, Oleg Belegradek and Oleg Bogopolsky for helpful discussion.

\section{References}

[1] V. Bardakov, V. Shpilrain and V. Tolstykh, 'On the palindromic and primitive width of a free group', J. Algebra 285 (2005), 574-585. 
[2] V. G. Bardakov, 'On the width of verbal subgroups of some free constructions', Algebra Logika 36 (1997), 494-517.

[3] G. Bergman, 'Generating infinite symmetric groups', Bull. London Math. Soc. 38 (2006), 429-440.

[4] I. V. Dobrynina, 'On the width in free products with amalgamation', Mat. Zametki 68 (2000), 353-359.

[5] V. Faiziev, 'A problem of expressibility in some amalgamated products of groups', J. Aust. Math. Soc. 71 (2001), 105-115.

[6] Yu. I. Merzlyakov, 'Algebraic linear groups as full groups of automorphisms and the closure of their verbal subgroups', Algebra Logika 6 (1967), 83-94.

[7] A. H. Rhemtulla, 'A problem of bounded expressibility in free products', Proc. Cambridge Philos. Soc. 64 (1969), 573-584.

Institute of Mathematics

Siberian Branch Russian Academy of Science 630090 Novosibirsk

Russia

e-mail: bardakov@math.nsc.ru
Department of Mathematics Yeditepe University 34755 Istanbul Turkey e-mail: vtolstykh@yeditepe.edu.tr 